\title{
A Subspace-Based Compensation Method for the Mutual Coupling in Concentric Circular Ring Arrays for Near-Field Source Localisation
}

\author{
Mohammed Jainul Abedin and Ananda Sanagavarapu Mohan \\ Centre for Health Technologies, School of Electrical, Mechanical and Mechatronic Systems, Faculty of Engineering and \\ Information Technology, University of Technology, Sydney (UTS), P.O. Box 123, Broadway, NSW 2007, Australia \\ Correspondence should be addressed to Ananda Sanagavarapu Mohan, ananda.sanagavarapu@uts.edu.au
}

Received 14 June 2011; Accepted 5 September 2011

Academic Editor: Hoi Shun Lui

Copyright ( $) 2012$ M. J. Abedin and A. S. Mohan. This is an open access article distributed under the Creative Commons Attribution License, which permits unrestricted use, distribution, and reproduction in any medium, provided the original work is properly cited.

\begin{abstract}
We propose a technique for compensating the effect of mutual coupling on parameter estimation that is suitable with any subspacebased super-resolution algorithms. A Concentric circular ring array (CCRA) formed using thin dipole antennas in the receiving mode is employed to estimate the parameters of electromagnetic sources located in the radiating near field of the array. A CCRA geometry that obtains a lowest Cramer-Rao lower bound (CRLB) in the presence of array mutual coupling is chosen for investigation. The mutual coupling among antenna elements of the array would affect the orthogonality of subspaces when MUSIC or ESPRIT algorithms are used for parameter estimation. The proposed method obtains a compensation matrix that restores the orthogonality between the subspaces there by improving the accuracy of estimation. To avoid three-dimensional searches, the range parameter is estimated using a cross-correlation-based method. Numerical simulation using a full-wave electromagnetic (EM) solver is employed to demonstrate the effectiveness of the proposed compensation approach.
\end{abstract}

\section{Introduction}

A common practical issue in parameter estimation using array of antennas is the effect of mutual coupling. This effect has long been recognised as one of the leading causes of degradation of the performance of parameter estimation algorithms [1]. This degradation occurs due to the presence of mutual coupling between antenna elements which causes deviations to the array manifold. Many methods to combat the effect of mutual coupling on parameter estimation have been proposed in the literature [1-4]. The concept of coupling matrix for compensating the effect of mutual coupling was used in [5]; however, it did not properly account for the platform effects. Full-wave EM solvers were also employed by many authors. Adve and Sarkar [6] used the method of moments for computing the mutual coupling effect precisely on wire antenna arrays, and Rogier and Zutter [7] used full-wave EM techniques for computing the mutual coupling effects in a planar array. A minimum norm mutual coupling compensation for the application of direction of arrival (DOA) estimation was also reported in [8]. Yuan et al. [9] proposed a method for DOA estimation using MUSIC algorithm by considering universal steering vectors which does not require any additional mutual coupling compensation method. In addition to the abovementioned methods, calibration techniques have also been proposed for compensating the effect of mutual coupling in DOA estimation algorithms. For instance, the calibration procedures developed by Weiss and Friedlander [10] require some perfectly calibrated sensors to compensate for the effect of mutual coupling as well as perturbations in gain and phase. An iterative least mean square approach was proposed by Hung [11] to estimate the calibration matrix, which requires some initial calibration. Self-calibration [12] and autocalibration [13] methods were also proposed which seek to iteratively minimise a function with respect to both direction of arrival (DOA) and mutual coupling. All the above-mentioned techniques mainly aim to compensate for 
the error due to lack of calibration, but all of them require a set of known calibration sources at some known locations. In practice, obtaining more than a single calibration source can be challenging for most of the localisation scenarios. Thus, compensation techniques that do not overly depend on multiple calibration sources are desirable. It should also be noted that all the above-mentioned works mainly aimed at either ULA or UCA geometries for compensating the mutual coupling. Mutual coupling matrix has a banded Toplitz structure for ULA and symmetric circulant structure with three bands for UCA [14], both of which can help to simplify the computation of the compensation matrix. However, such well-defined matrix structure may not be available for most of the complex arbitrary array geometries such as concentric circular ring arrays (CCRA) for which the effect of mutual coupling can be more severe. It would, therefore, be of interest to develop alternate mutual coupling compensation methods useful for complex planar arrays, which is the focus of this paper.

The effect of mutual coupling varies in arrays since the mutual impedances in a transmitting array can be different from that of a receiving array. Lui et al. $[15,16]$ have analysed this effect especially for the receiving mode of antenna arrays. They also propose a mutual coupling compensation technique based on a least square approach for the receiving mode of antenna arrays $[15,17]$. However, their compensation technique can suffer from performance degradation when the number of emitting sources is less than the number of elements in the receiver antenna array. This is mainly because the compensation technique based on least squares method does not have a unique solution when either a single source or a smaller number of sources are present. Further, the investigations reported in $[15,17]$ on mutual coupling compensation are carried out only for ULA and UCA geometries for the estimation of azimuth angles of farfield sources. However, in many applications, estimation of the parameters of near-field sources when complex arbitrary antenna arrays are employed is of concern and particularly the techniques to reduce the effects of mutual coupling on the parameter estimation are of interest.

For near-field parameter estimation, the use of planar arrays can be more appropriate to overcome the limitations of ULAs, namely, the problems in resolving the noncoplanar emitters and the presence of angular ambiguities within the azimuthal plane [18]. However, the use of complex planar array geometries can pose some extra challenges in the form of the array steering vector imperfections due to mutual coupling which can lead to severe detrimental effects. Further, when parameters need to be estimated, the effect of mutual coupling varies on the type of estimation algorithms used. For subspace-based super-resolution estimation algorithms, the error due to mutual coupling can rotate the subspaces thereby disrupting the orthogonality between signal and noise subspaces. As a result, subspace-based super-resolution algorithms such as MUSIC and ESPRIT would lead to significant degradation of their estimation performance. These errors must be compensated in order to recover their estimation performance. Hence, there is a need for mutual coupling compensation methods to improve the estimation performance of MUSIC and ESPRIT algorithms for localising either single or multiple near-field sources when arbitrary planar antenna arrays are employed.

In this paper, we propose a mutual coupling compensation technique applicable for MUSIC and ESPRIT algorithms and investigate its performance when it is applied on a CCRA for the near-field parameter estimation. CCRA geometry can be formed by having rings that have either uniform or nonuniform radii. Similarly, the number of antenna elements over the circumference of each ring could also be variable. All these variations have an impact on the array mutual coupling of CCRA which in turn affects the parameter estimation. Thus, in order to choose an optimised CCRA geometry that can lead to lowest estimation bias; we derive CRLB for four different cases each with different ring radii and number of antenna elements so as to obtain a particular combination which provides the lowest root mean square error. The optimised array geometry is then employed for further processing, that is, the mutual coupling compensation and nearfield parameter estimation using both MUSIC and ESPRIT algorithms. Since the parameter set required to be estimated for near-field localisation is larger than for the corresponding far-field case, it would be useful to reduce the computational load of conventional MUSIC and ESPRIT algorithms. To achieve this objective, here the range parameter is estimated using a cross-correlation-based method. Further, beamspace processing [19] is applied on CCRA so as to make it suitable for use with MUSIC and ESPRIT algorithms. We assume that the antennas receive correlated signals. Since the beamspace processing maps the array steering manifold of a planar array onto that of a virtual linear array, forward-backward smoothing can also be applied for decorrelation [18]. We employ a three-ring CCRA with thin wire dipole antennas as elements and obtain the mutual coupling matrix using a full-wave electromagnetic simulator. Simulation results are provided on the 3D localization of radiating sources placed in the near field of the CCRA by incorporating the proposed mutual coupling compensation method on MUSIC and ESPRIT algorithms.

\section{Problem Formulation}

2.1. Concentric Circular Ring Array (CCRA). A CCRA is designed with $P$ concentric rings, and each ring is populated with the same number of $M$ omnidirectional dipole antennas on its circumference, as shown in Figure 1. The location of $k$ th element on $l$ th ring is denoted by its Cartesian coordinate $\left\{x_{k_{l}}, y_{k_{l}}\right\}=\left\{\rho_{l} \cos \varphi_{k_{l}}, \rho_{l} \sin \varphi_{k_{l}}\right\}$, where $k_{l}=$ $1_{1}, 2_{1}, \ldots, M_{1}, 1_{2}, 2_{2}, \ldots, M_{2}, \ldots, M_{P}$, with respect to the origin chosen at the centre of the circular ring structure, where $\rho_{l}$ is the radius of the $l$ th ring and $\varphi_{k_{l}}$ is the angular position of the $k$ th element of the $l$ th ring. For the uniform placement of array elements on the circumference of each ring, $\varphi_{k_{l}}=$ $2 \pi\left(k_{l}-1\right) / M_{P}$. The radius of the $l$ th ring is given by

$$
\rho_{l}=\frac{\lambda_{\min }}{\left(4 \sin \left(\pi / M_{P}\right)\right)},
$$




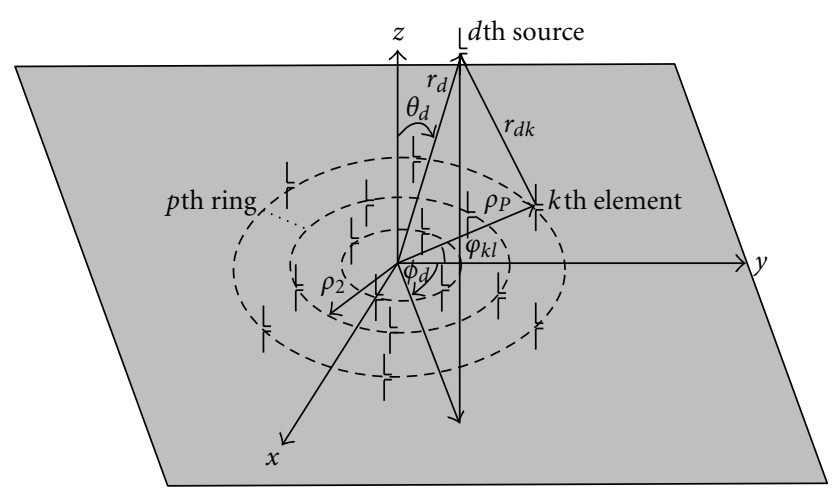

Figure 1: Concentric circular ring array (CCRA).

where $\lambda$ is the wavelength. Now, the elements of the array steering vector for the $l$ th ring can be obtained as

$$
a_{k_{l}}=e^{j \omega \tau_{k_{l}}(r, \theta, \phi)},
$$

where $\tau_{k_{l}}$ is the time delay of incoming signal coming from a source located at $(r, \theta, \phi)$ to $k$ th element of $l$ th ring, and time delay is determined as

$$
\tau_{k_{l}}(r, \theta, \phi)=\frac{1}{c}\left(r_{k_{l}}-r\right)
$$

where

$$
r_{k_{l}}=\operatorname{sqrt}\left(r^{2}+\left(x_{k_{l}}^{2}+y_{k_{l}}^{2}\right)^{2}-2 \sin \theta\left(x_{k_{l}} \cos \phi+y_{k_{l}} \sin \phi\right)\right) \text {. }
$$

The array steering vector for the $l$ th ring is given by

$$
\mathbf{a}_{l}=\left[e^{j \omega \tau_{1_{l}}(r, \theta, \phi)}, e^{j \omega \tau_{2_{l}}(r, \theta, \phi)}, \ldots, e^{j \omega \tau_{M_{l}}(r, \theta, \phi)}\right]^{T},
$$

where $[\cdot]^{T}$ denotes transpose operation. Considering all the rings in the CCRA, the array steering vector can be written as

$$
\mathbf{a}(r, \theta, \phi)=\left[\mathbf{a}_{1}(r, \theta, \phi), \mathbf{a}_{2}(r, \theta, \phi), \ldots, \mathbf{a}_{P}(r, \theta, \phi)\right]^{T} .
$$

A CCRA can be formed either by having same or varying number of elements on each ring [20]. Hence, the array steering vector of every ring may not be equal in length and thus can be represented as a group of separate single array steering vectors. The dimension of the total array steering vector is $M P \times 1$, given by

$$
\mathbf{a}(r, \theta, \phi)=\left[\begin{array}{c}
e^{j \omega \tau_{1_{1}}(r, \theta, \phi)}, e^{j \omega \tau_{2_{1}}(r, \theta, \phi)}, \ldots, e^{j \omega \tau_{M_{1}}(r, \theta, \phi)}, e^{j \omega \tau_{1_{2}}(r, \theta, \phi)}, \\
e^{j \omega \tau_{2_{2}}(r, \theta, \phi)}, \ldots, e^{j \omega \tau_{M_{2}}(r, \theta, \phi)}, e^{j \omega \tau_{1_{l}}(r, \theta, \phi)}, e^{j \omega \tau_{2_{l}}(r, \theta, \phi)}, \ldots, e^{j \omega \tau_{M_{P}}(r, \theta, \phi)}
\end{array}\right]^{T}
$$

With an aim to apply beamspace transformation, we express the array steering vector of CCRA into a phase mode as

$$
\mathbf{a}_{f}=f_{p}^{H} \mathbf{a}(r, \theta, \phi) .
$$

This transformation maps the array steering vector of CCRA to that of an equivalent virtual linear array. Defining "B" as the discrete Fourier transform matrix given by

$$
\begin{aligned}
& \mathbf{B}=\left[\begin{array}{cccc}
1 & e^{-j h \varphi} & \cdots & e^{-j h(M-1) \varphi} \\
\vdots & \vdots & \vdots & \vdots \\
1 & e^{j h \varphi} & \cdots & e^{j h(M-1) \varphi}
\end{array}\right] \\
& \mathbf{B}=\left[\begin{array}{c}
f_{-h}^{H} \\
\vdots \\
f_{h}^{H}
\end{array}\right],
\end{aligned}
$$

where $(\cdot)^{H}$ denotes complex conjugate transpose and the elements of (9) can be expressed as

$$
f_{p}=\frac{1}{M}\left[1, e^{-j p \varphi}, \ldots, e^{-j p(M-1) \varphi}\right]^{T},
$$

where $p=-h,-h+1, \ldots, h$.
2.2. Selection of Optimal CCRA Configuration Based on CRLB. Here, our aim is to select an optimal CCRA configuration that provides lowest estimation bias using Cramer-Rao lower bound (CRLB) and employ that array for further processing in this paper. CRLB is derived in this section for CCRA in the presence of mutual coupling. Assuming the received signal vector $\mathbf{s}$ to be complex valued and Gaussian distributed, the covariance matrix can be formed as

$$
\mathbf{R}=\overline{\mathbf{A}} \mathbf{R}_{s} \overline{\mathbf{A}}^{H}+\sigma_{n}^{2} \mathbf{I},
$$

where $\overline{\mathbf{A}}=\mathbf{C A}$ and $\mathbf{R}_{s}=E\left[\mathbf{s s}^{H}\right]$. The unknown parameters which need to be estimated can be written in a vector form

$$
\Theta=\left[\mathbf{r}, \boldsymbol{\theta}, \boldsymbol{\phi},\left[\alpha_{k}\right]_{1: M P},\left[\beta_{k}\right]_{1: M P}\right]^{T},
$$

where $\boldsymbol{\theta}=\left[\theta_{1}, \ldots, \theta_{d}\right]^{T}, \boldsymbol{\phi}=\left[\phi_{1}, \ldots, \phi_{d}\right]^{T}, \alpha_{k}=\left\{C_{k}^{\mathrm{re}}\right\}$, and $\beta_{k}=\left\{C_{k}^{\text {im }}\right\}$. For convenience, we are using notations $\alpha_{k}$ and $\beta_{k}$ instead of $\alpha_{k_{l}}$ and $\beta_{k_{l}}$, for $k=1,2, \ldots, M P$. Considering $N$ snapshots of the received signal, the Fisher Information Matrix (FIM) can be formed as

$$
F_{m n}=N \operatorname{tr}\left\{R^{-1} \frac{\partial R}{\partial \Theta_{m}} R^{-1} \frac{\partial R}{\partial \Theta_{n}}\right\}
$$


The elements of FIM are derived [21,22] and presented in the appendix. Referring to the appendix, the FIM which is a $(3 D+M P) \times(3 D+M P)$ matrix can be written as

$$
\mathrm{FIM} \triangleq \mathbf{F}=\left|\begin{array}{lllll}
F_{\theta \theta} & F_{\theta \phi} & F_{\theta r} & F_{\theta \alpha} & F_{\theta \beta} \\
F_{\theta \phi}^{T} & F_{\phi \phi} & F_{\phi r} & F_{\phi \alpha} & F_{\phi \beta} \\
F_{\theta r}^{T} & F_{r \phi}^{T} & F_{r r} & F_{r \alpha} & F_{r \beta} \\
F_{\theta \alpha}^{T} & F_{\alpha \phi}^{T} & F_{r \alpha}^{T} & F_{\alpha \alpha} & F_{\alpha \beta} \\
F_{\theta \beta}^{T} & F_{\phi \beta}^{T} & F_{r \beta}^{T} & F_{\beta \alpha} & F_{\beta \beta}
\end{array}\right|,
$$

where each term represents a block matrix, for example, $F_{\theta \alpha}=\left[F_{\theta \alpha_{k}}\right]_{k=1: M P}, F_{\alpha \boldsymbol{\alpha}}=\left[F_{\alpha_{k} \alpha_{k}}\right]_{k=1: M P}$, and $F_{\beta \beta}=$ $\left[F_{\beta_{k} \beta_{k}}\right]_{k=1: M P}$ and the other block matrices follow the same notational convention. CRLB is the inverse of FIM given by $\mathbf{J}=\mathbf{F}^{-1}$, and for all the desired parameters it can be obtained as

$$
\begin{gathered}
\operatorname{CRLB}_{\Theta}=\operatorname{sqrt}\left(\frac{1}{2 D} \sum_{d=1}^{3 D} J_{d d}\right) \\
\mathrm{CRLB}_{c}=\operatorname{sqrt}\left(\frac{1}{\|c\|^{2}} \sum_{d=3 D+1}^{3 D+M P} J_{d d}\right) \times 100 \% .
\end{gathered}
$$

In (16), CRLB with mutual coupling is calculated as a percentage as it is a relative value [21]. The CRLB for CCRA is computed by varying either the number of antenna elements or the ring radii, so that we can chose an optimum array configuration that offers the lowest bias for estimating the near-field parameters in the presence of array mutual coupling. In this paper, we consider four different variations of CCRA for computing the CRLB and the results are indicated in Figures 2, 3, and 4. It can be observed, from Figures 2-4, that lowest CRLB results when the number of antenna elements on each ring is not the same and also when the incremental variation of ring radii is not uniform. Thus, the optimal CCRA configuration for performing the nearfield parameter estimation is chosen.

2.3. Signal Modelling in the Presence of Mutual Coupling. The array response of an $M$-element CCRA due to $d$ th near-field source located at $(r, \theta, \phi)$ is given by

$$
x(t)=\sum_{d=1}^{D} \mathbf{C a}_{f}\left(r_{d}, \theta_{d}, \phi_{d}\right) s_{d}(t)+n(t)
$$

where $\mathbf{C}$ denotes a matrix that contains the mutual coupling among the array elements. The coupling is inversely proportional to the distance between the elements. After normalising the main diagonal elements to unity, the mutual coupling matrix is expressed as follows:

$$
\mathbf{C}=\left(Z_{A}+Z_{T}\right)\left(\mathbf{Z}+Z_{T} \mathbf{I}_{N}\right)^{-1},
$$

where $Z_{A}$ is the impedance of an isolated individual element, $Z_{T}$ is the impedance of each receiver element, and $\mathbf{I}_{M}$ is the identity matrix. The impedance $Z_{T}$ is considered to be the

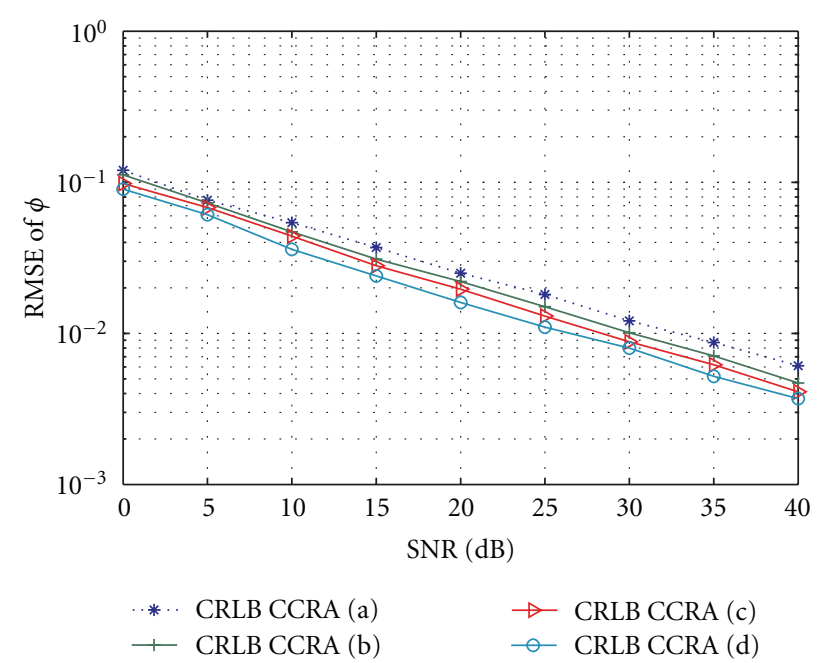

FIgURE 2: CRLB of estimated azimuth for four different arrangements: (a), (b), (c), and (d) of CCRA.

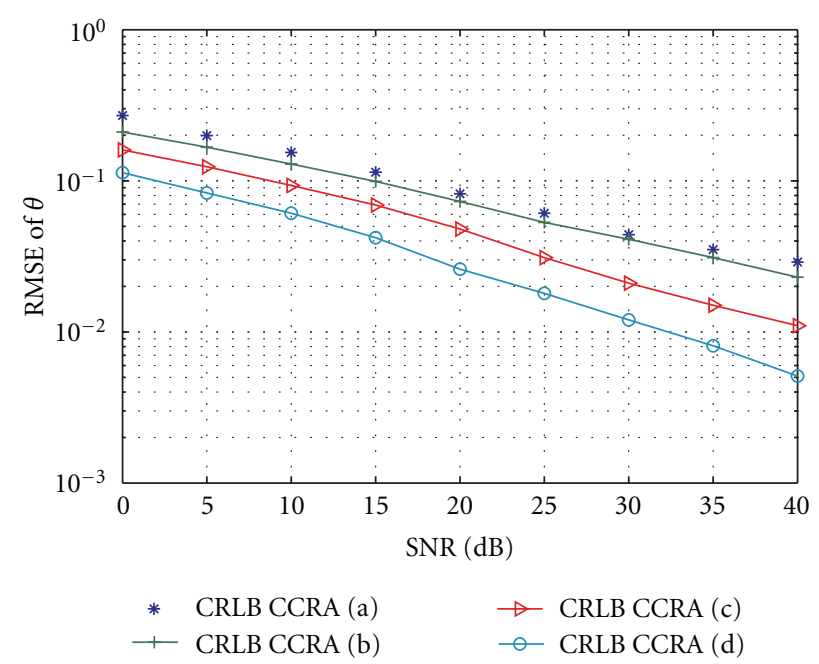

Figure 3: CRLB of estimated elevation angle for four different arrangements: (a), (b), (c), and (d) of CCRA.

complex conjugate of $Z_{A}$ ensuring impedance matching for maximum power transfer. The elements of $\mathbf{Z}$ matrix are

$$
\mathbf{Z}=\left|\begin{array}{cccc}
Z_{A}+Z_{T} & Z_{1_{1} 2_{1}} & \cdots & Z_{1_{1} M_{P}} \\
Z_{2_{1} 1_{1}} & Z_{A}+Z_{T} & \cdots & Z_{2_{1} M_{P}} \\
\vdots & \vdots & \vdots & \vdots \\
Z_{M_{P} 1_{1}} & Z_{M_{P} 2_{1}} & \cdots & Z_{A}+Z_{T}
\end{array}\right| .
$$

Now, $Z$ is defined as

$$
Z=\left[\left\{z_{k_{l} k_{l}}\right\}\right]=\left[\left\{R_{k_{l} k_{l}}+j X_{k_{l} k_{l}}\right\}\right]
$$

where $R_{k_{l} k_{l}}$ and $X_{k_{l} k_{l}}$ are the resistive and reactive components of the impedance $Z$. Using a number of snapshots of the 


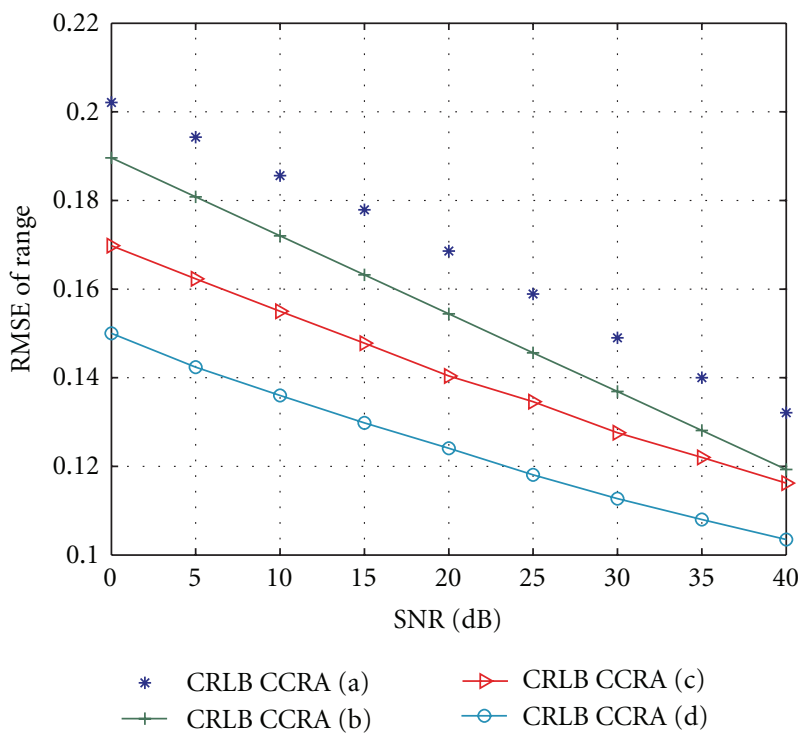

FIGURE 4: CRLB of estimated range for four different arrangements: (a), (b), (c), and (d) of CCRA.

received signal in (17), the data covariance matrix can be expressed as

$$
\mathbf{R}=\sum_{d=1}^{D} \sigma_{d}^{2} \mathbf{C a}_{f}\left(r_{d}, \theta_{d}, \phi_{d}\right) \mathbf{a}_{f}^{H}\left(r_{d}, \theta_{d}, \phi_{d}\right) \mathbf{C}^{H}+\sigma_{n}^{2} \mathbf{I} .
$$

In order to apply subspace-based algorithms such as MUSIC or ESPRIT for parameter estimation, an eigenvalue decomposition has to be performed which is given by

$$
\mathbf{R}=\mathbf{U}_{s} \Lambda_{s} \mathbf{U}_{s}^{H}+\sigma_{n}^{2} \mathbf{U}_{n} \mathbf{U}_{n}^{H},
$$

where $\mathbf{U}_{s}$ and $\mathbf{U}_{n}$ contain the signal and noise eigenvectors, $\Lambda_{s}$ is the diagonal matrix that contains signal power, and $\sigma_{n}^{2}$ denotes noise power. In order to estimate parameters accurately, the signal and noise subspaces must be mutually orthogonal. But, in the presence of mutual coupling C, the orthogonality no longer exists between signal and noise subspaces. Hence, we need to obtain a matrix $\widetilde{\mathbf{C}}$ that can compensate the effects of mutual coupling and thereby restoring orthogonality between the subspaces. Thus, incorporation of $\widetilde{\mathbf{C}}$ makes $\left\|\mathbf{U}_{n}^{H} \widetilde{\mathbf{C}} \mathbf{a}\left(r_{d}, \theta_{d}, \phi_{d}\right)\right\|=0$ for $d=1, \ldots, D$ which will lead to proper estimation of the parameters using subspace-based algorithms. However, in practice, $\widetilde{\mathbf{C}}$ is not known. Hence, methods are required to accurately estimate $\widetilde{\mathbf{C}}$ prior to applying any subspace-based parameter estimation procedure when the mutual coupling is present among the elements of the array.

2.4. Subspace Rotation due to Array Mutual Coupling. For the CCRA, in an ideal case, that is, when the mutual coupling is ignored, the array steering vector due to an incident signal from $d$ th source is given by (8). Under the effect of mutual coupling, the modified array steering vector $\widetilde{\mathbf{a}}_{f}(r, \theta, \phi)$ is given by

$$
\widetilde{\mathbf{a}}_{f}(r, \theta, \phi)=\mathbf{C a}_{f}(r, \theta, \phi) .
$$

As noted earlier, the mutual coupling disrupts the orthogonality of subspaces since the signal subspace that is spanned by the steering vector is rotated from its true position. Therefore, it can be seen that

$$
\operatorname{span}\left\{\tilde{A}_{f}\right\}=\operatorname{span}\left\{\mathbf{C} A_{f}\right\}
$$

where $A_{f}$ is the array manifold matrix of size $M P \times D$ that is created from the array steering vector (7) in the presence of $D$ sources. Referring to (17), the mutual coupling matrix C for CCRA is given by

$$
\mathbf{C}=\left|\begin{array}{ccccccccccc}
c_{1_{1} 1_{1}} & c_{1_{1} 2_{1}} & c_{1_{1} 3_{1}} & \cdots & c_{1_{1} M_{1}} & c_{1_{1} 1_{2}} & c_{1_{1} 2_{2}} & \cdots & c_{1_{1} k_{l}} & \cdots & c_{1_{1} M_{P}} \\
c_{2_{1} 1_{1}} & c_{21} 2_{1} & c_{21_{1}} & \cdots & c_{2_{1} M_{1}} & c_{2_{1} 1_{2}} & c_{2_{1} 2_{2}} & \cdots & c_{2_{1} k_{l}} & \cdots & c_{2_{1} M_{P}} \\
\vdots & \vdots & \vdots & & \vdots & \vdots & \vdots & & \vdots & & \vdots \\
c_{1_{2} 1_{1}} & & & & & & & & & & \\
\vdots & & & & & & & & & & \\
c_{1_{p} 1_{1}} & & & & c_{1_{p} M_{1}} & & & & c_{1_{p} k_{l}} & \\
\vdots & & & \ddots & & & & \ddots & & \ddots & \\
c_{M_{p} 1_{1}} & c_{M_{p} 2_{1}} & \cdots & & c_{M_{p} M_{1}} & & \cdots & & c_{k_{p} k_{l}} & \cdots & c_{M_{p} M_{p}}
\end{array}\right| .
$$

Now, the rotated subspace due to $\mathbf{C}$ can be expressed as

$$
\widetilde{\mathbf{u}}(r, \theta, \phi)=\mathbf{C u}(r, \theta, \phi),
$$

where $\tilde{\mathbf{u}}$ are the elements of the rotated signal and noise subspaces due to the presence of mutual coupling. The effect of mutual coupling $\mathbf{C}$ must be compensated so as to make subspaces orthogonal in order to accurately estimate parameters. 
2.5. Subspace Rotation due to Simplified Mutual Coupling Model in a CCRA. Our aim here is to derive the rotated subspaces for CCRA under simplified assumptions to demonstrate our method. The mutual coupling in an array depends on the separation between two adjacent antenna elements, and hence nonadjacent array elements have weaker coupling. Realising this, a single coupling coefficient was considered for computing the compensation matrix in the literature $[14,23]$. Also, the coupling effect was ignored for an ULA for interelement separation greater than $0.707 \lambda$ [21]. In this section, in order to derive the subspace rotation due to mutual coupling, we simplify (25) by assuming large interring separation for CCRA so as to make the interring coupling insignificant and thus can be ignored. Also, in this case, coupling among elements of outer ring can also be ignored due to larger interelement separation since the elements are distributed uniformly on rings with larger circumferences thus leading to larger interelement separation. Under these assumptions, only the mutual coupling among the adjacent elements of the innermost rings will be considered to simplify the problem. Thus, the simplified mutual coupling matrix $\mathbf{C}$ can be obtained from (25) as

$$
\mathbf{C}=\left[\begin{array}{ccccc}
1 & c_{1_{1}} & c_{2_{1}} & \cdots & 0 \\
c_{1_{1}} & 1 & c_{1_{1}} & \cdots & 0 \\
\vdots & & \ddots & & \vdots \\
0 & 0 & & \cdots & 1
\end{array}\right]
$$

Now, simplifying (25) for the above case, the expression for the rotated subspace can be expressed as

$$
\begin{aligned}
& \tilde{\mathbf{u}}(r, \theta, \phi)=\left[1+c_{1_{1}} u, c_{1_{1}}+a+c_{2_{1}} u^{2}, \ldots, c_{2_{1}} u^{M-3}+u^{M-2}\right. \\
& \left.+c_{2_{1}} u^{M-1}, c_{2_{1}} u^{M-2}+u^{M-1}\right]^{T} \text {. }
\end{aligned}
$$

After further manipulation, (28) can be represented as

$$
\begin{aligned}
\tilde{\mathbf{u}}(r, \theta, \phi)= & \left(1+c_{2_{1}} u\right)\left[1, u, \ldots, u^{M-2}, u^{M-1}\right]^{T} \\
& +c_{2_{1}}\left[0,1, \ldots, u^{M-3}, u^{M-2}-u^{M}\right]^{T} \\
= & \mathbf{u}_{m c}^{1}(r, \theta, \phi)+\mathbf{u}_{m c}^{2}(r, \theta, \phi),
\end{aligned}
$$

where $\mathbf{u}_{m c}$ denotes the modified eigenvector in the presence of mutual coupling. Thus, the expression for the estimated covariance matrix modified due to the presence of mutual coupling becomes

$$
\begin{aligned}
\mathbf{R}= & \mathbf{U}_{m c}^{1}{ }^{H}\left(r_{d}, \theta_{d}, \phi_{d}\right) \Lambda_{s} \mathbf{U}_{m c}^{1}\left(r_{d}, \theta_{d}, \phi_{d}\right) \\
& +\mathbf{U}_{m c}^{2}{ }^{H}\left(r_{d}, \theta_{d}, \phi_{d}\right) \Lambda_{s} \mathbf{U}_{m c}^{2}\left(r_{d}, \theta_{d}, \phi_{d}\right)+\sigma_{n}^{2} \mathbf{I} .
\end{aligned}
$$

Comparing (30) and (21), for the simplified case considered here, one can observe that the variation in covariance matrix is due to the contribution of the factor $1+c_{2_{1}} u$ which causes subspaces to rotate. As a result, it would lead to performance degradation of the subspace-based superresolution algorithms.

\section{Compensation for the Mutual Coupling}

The effect of mutual coupling must be compensated for achieving a desired performance. This can be achieved by formulating a compensation matrix that minimises the error by which the array manifold gets deviated from that of actual one [14]. For subspace-based estimation algorithms, the deviation in array manifold due to mutual coupling does appear as an error that disrupts the orthogonality between signal and noise subspaces of the covariance matrix. Similar concept was also used in [24] where genetic algorithm (GA) was used to minimise the error to obtain a compensation matrix in order to recover the estimation performance of the MUSIC algorithm. However, the method proposed in [24] requires MUSIC pseudospectrum for a known calibration source to correlate with the pseudospectra of unknown sources at each search point in the space which can be computationally demanding. Alternatively, in this paper, we propose a method that uses an ideal array response without mutual coupling to estimate a compensation matrix by minimising the error between the rotated and ideal subspaces, so that the introduction of the compensation matrix restores the orthogonality between the subspaces. The advantage of the method proposed here as compared to that used in [24] is that, in the present case, the compensation matrix can be determined with a lower computational load as it does not require performing correlation of MUSIC pseudospectra at every search point within the parameter space. As a result, the proposed compensation method achieves quicker convergence. Also, the proposed method is applicable for any subspace-based parameter estimation algorithm, as will be demonstrated below where we also provide a detailed description of the proposed technique.

3.1. Role of the Compensation Matrix. In order to remove the effect of mutual coupling completely, one needs to estimate the compensation matrix as $\widetilde{\mathbf{C}}=\mathbf{C}^{-1}$. However, in actual practice, the array output model cannot account for all the sources of signal impairments in an antenna array, hence it is difficult to achieve $\widetilde{\mathbf{C}}$ equal to $\mathbf{C}^{-1}$. Considering (17), even in a noise-free situation, that is, when $\mathbf{n}(t)$ becomes a null vector, $\mathbf{C}^{-1}$ can only be considered to be proportional to $\widetilde{\mathbf{C}}$. Therefore, only an estimated compensation matrix can be obtained. Assuming that ideal array response from reference sources is known, the estimation procedure for $\widetilde{\mathbf{C}}$ can be expressed as a minimisation of the function which is given by

$$
\min _{\widetilde{\mathbf{C}}}\left\|\mathbf{a}_{k_{l}}^{H}(r, \theta, \phi) \tilde{\mathbf{C}} \mathbf{R}_{x} \tilde{\mathbf{C}}^{H} \mathbf{a}_{k_{l}}(r, \theta, \phi)\right\|_{F}^{2},
$$

where $\mathbf{a}_{k_{l}}(r, \theta, \phi)$ is the orthogonal null steering vector that spans the equivalent null space of the reference source. Incorporating $\widetilde{\mathbf{C}}$ obtained from (31) into (26), the subspace $\widetilde{\mathbf{U}}$ will approximately be equal to $\mathbf{U}$ since $\mathbf{C} \widetilde{\mathbf{C}} \approx \mathbf{I}$. Also, in (28), all coupling terms will almost vanish once the compensation terms are incorporated and thereby the subspace rotation due to the effect of mutual coupling will 
almost be nullified resulting in the effective restoration of orthogonality between subspaces.

Comparing the proposed technique with the other published compensation techniques in the literature shows that the method used in $[11,15]$ requires at least $M P$ different near-field calibration sources around CCRA to achieve $\mathbf{a}_{k_{l}}(r, \theta, \phi)$ of (31), where $k_{l}=1_{1}, 2_{1}, \ldots, M_{1}, 1_{2}, 2_{2}, \ldots$, $M_{2}, \ldots, M_{P}$. This requires estimation of a compensation matrix $\widetilde{\mathbf{C}}$ of the size $M P \times M P$ by minimising (31) for all $M P$ calibration sources. For the same case, the least square approach would require at least more than $M P$ calibration sources to achieve a unique solution for the estimation of compensation matrix $\widetilde{\mathbf{C}}$. Therefore, methods that do not require multiple calibration sources for estimating the compensation matrix are desirable.

3.2. Proposed Compensation Method. Here, it is hypothesized that the error that destroys the orthogonality between signal and noise subspaces is only due to the mutual coupling and all other possible sources of impairment are ignored. As a result, $\tilde{\mathbf{U}}_{n}^{H} A(r, \theta, \phi) \neq 0$, which is due to the presence of mutual coupling among the elements of the receiver antenna array. Here, $\tilde{\mathbf{U}}_{n}$ is the noise subspace that is obtained from EVD of covariance matrix formed by the measured data at the receiver. Since $\tilde{\mathbf{U}}_{n}$ is corrupted by the array mutual coupling, we need to estimate $\widetilde{\mathbf{C}}$. For that, instead of minimising the functional given in (31), we reformulate an objective function $Q$ as

$$
Q=\min _{\widetilde{\mathbf{C}}}\left\|\tilde{\mathbf{U}}_{n}^{H} A(r, \theta, \phi)-\mathbf{U}_{n}^{H} \widetilde{\mathbf{C}} A(r, \theta, \phi)\right\|_{F}^{2} .
$$

Now, the estimated compensation matrix $\widetilde{\mathbf{C}}$ can be computed by performing an optimisation method to minimise (32). Here, the true noise subspace $\mathbf{U}_{n}$ is obtained from a single known near-field calibration source for an ideal array without mutual coupling with known array geometry and element positions and assuming no gain and phase deviation to occur. We employ genetic algorithm to determine $\widetilde{\mathbf{C}}$ by minimising $Q$ of (32). Incorporating $\widetilde{\mathbf{C}}$ restores the orthogonality between subspaces, thereby recovering the performance of parameter estimation even in the presence of array mutual coupling.

\section{Parameter Estimation after Compensation}

In this section, we show as to how the proposed technique of compensation is incorporated into both MUSIC and ESPRIT algorithms for estimating the parameters of nearfield sources. In order to reduce the computational burden in MUSIC and ESPRIT, we estimate range parameter by using a method that employs cross-correlation among the eigenvectors of the signal subspace [25]. A brief description of the procedure for parameter estimation of near-field sources is given below.

4.1. MUSIC Algorithm for Estimating Azimuth and Elevation Angles. The azimuth and elevation angles of near-field sources can be obtained from the two-dimensional MUSIC pseudospectrum given by

$$
P=\frac{1}{\left\|\mathbf{U}_{n} \tilde{\mathbf{C}} \mathbf{a}_{f}(\theta, \phi)\right\|^{2}},
$$

where $\widetilde{\mathbf{C}}$ is the estimated compensation matrix obtained by minimising $Q$ in (32).

4.2. ESPRIT Algorithm for Estimating Azimuth and Elevation Angles. For ESPRIT algorithm, the signal subspace matrices can be written as [26]

$$
\Gamma \mathbf{U}=\mathbf{U}^{-1} \Psi+\mathrm{DIU}^{-1} \Psi^{*},
$$

where $\Psi=T^{-1} \Phi T, \mathbf{D}=\operatorname{diag}\left\{(-1)^{M_{P}-2}, \ldots,(-1)^{1},(-1)^{0}\right.$, $\left.(-1)^{1}, \ldots,(-1)^{M_{P}}\right\}$ and $\mathbf{U}=\widetilde{\mathbf{U}} \widetilde{\mathbf{C}}$ is the matrix of eigenvectors that is obtained after mutual coupling compensation. This system of equations has a unique solution when the number of sources is less than the number of antenna elements in CCRA. The solution can be obtained as $\Phi=T \Psi T^{-1}$, and the eigenvalues of $\Psi$ provide the diagonal elements of $\Phi$ which therefore yield automatically paired source azimuth and elevation angles as

$$
\begin{gathered}
\phi_{d}=\arg \left(u_{d}+j v_{d}\right), \\
\theta_{d}=\sin ^{-1}\left(\operatorname{sqrt}\left(u_{d}^{2}+v_{d}^{2}\right)\right) .
\end{gathered}
$$

4.3. Cross-Correlation among Signal Eigenvectors for TimeDelay Estimation. Since the eigenvectors contain the time delay information, the time delay among the signal paths can be estimated via the cross-correlation of eigenvectors. The compensated signal eigenvectors are $\mathbf{u}_{i}$ and $\mathbf{u}_{j}$, where $\mathbf{u}_{i}=\widetilde{\mathbf{C}} \widetilde{\mathbf{u}}_{i}$ and $\mathbf{u}_{j}=\widetilde{\mathbf{C}} \tilde{\mathbf{u}}_{j}$. A normalised cross-correlation of $\mathbf{u}_{i}$ and $\mathbf{u}_{j}$ is given by

$$
R_{i, j}(\tau)=\frac{r_{i, j}(\tau)}{\sqrt{r_{i, i}(0) r_{j, j}(0)}},
$$

where the $r_{i, j}(\tau)$ is the cross-correlation of $\mathbf{u}_{i}\left(k_{l}\right)$ and $\mathbf{u}_{j}\left(k_{l}\right)$ and it can be expressed as

$$
r_{i, j}(\tau)=\frac{1}{N_{s}-\tau} \sum_{k_{l}=1}^{M_{P}-\tau} u_{i}^{*}\left(k_{l}+\tau\right) u_{j}\left(k_{l}\right),
$$

where $\tau=-M_{P}+1, \ldots, M_{P}-1$ and $u_{i}(n)$ is the $n$th element of $\mathbf{u}$. An estimated time delay can be obtained by maximising the $R_{i, j}(\tau)$. Using the time delay, the range parameter is estimated.

\section{Simulation Results and Discussion}

In order to demonstrate the performance of the proposed compensation method for near-field source localisation, we consider a CCRA with 27 half-wave, thin, dipole antennas which are distributed uniformly along the circumferences 
TABLE 1: The different CCRA configurations used for obtaining the optimal case.

\begin{tabular}{lcr}
\hline CCRA configurations & Radii of rings & No. of elements on each ring \\
\hline (a) & $\rho_{1}=0.5 \lambda, \rho_{2}=\lambda$, and $\rho_{3}=1.5 \lambda$ & $M_{1}=9, M_{2}=9$, and $M_{3}=9$ \\
(b) & $\rho_{1}=0.5 \lambda, \rho_{2}=1.2 \lambda$, and $\rho_{3}=2 \lambda$ & $M_{1}=9, M_{2}=9$, and $M_{3}=9$ \\
(c) & $\rho_{1}=0.5 \lambda, \rho 2=\lambda$, and $\rho 3=1.5 \lambda$ & $M_{1}=7, M_{2}=9$, and $M_{3}=11$ \\
(d) & $\rho_{1}=0.5 \lambda, \rho_{2}=1.2 \lambda$, and $\rho_{3}=2 \lambda$ & $M_{1}=7, M_{2}=9$, and $M_{3}=11$ \\
\hline
\end{tabular}

TABLE 2: Values of estimated compensation matrix using the proposed method.

\begin{tabular}{|c|c|c|}
\hline$\tilde{c}_{1_{1} 1_{1}}=1.0000000000000+0.0000000000000 i$ & $\begin{array}{l}\tilde{c}_{1_{1} 1_{2}}= \\
-16.4480117045500+14.6902890526432 i\end{array}$ & $\begin{array}{l}\tilde{c}_{1_{1} 1_{3}}= \\
-16.4480117045500+14.6902890526432 i\end{array}$ \\
\hline$\widetilde{\mathcal{c}}_{1_{12}{ }_{1}}=-5.96190733331502-35.7977458691057 i$ & $\begin{array}{l}\tilde{\mathcal{c}}_{1_{1} 2_{2}}= \\
8.64950808461148-10.6569065324454 i\end{array}$ & $\begin{array}{l}\tilde{c}_{1_{1} 2_{3}}= \\
15.3736986943575+0.998396037519312 i\end{array}$ \\
\hline$\tilde{c}_{l_{1} 3_{1}}=8.23261772059128+15.7095769032906 i$ & $\begin{array}{l}\tilde{c}_{1_{1} 3_{2}}= \\
-3.78253875856304+9.10367969640321 i\end{array}$ & $\begin{array}{l}\tilde{c}_{1_{1} 3_{3}}= \\
-7.48101029224301-9.22054742279721 i\end{array}$ \\
\hline$\tilde{c}_{1_{1} 4_{1}}=46.7641650534059-30.5168801643121 i$ & $\begin{array}{l}\tilde{c}_{1_{1} 4_{2}}= \\
-1.84838342545929+19.3049333382765 i\end{array}$ & $\begin{array}{l}\tilde{\mathcal{c}}_{1_{1} 4_{3}}= \\
-23.2217879987250-18.535361202\end{array}$ \\
\hline$\tilde{c}_{1_{1} 5_{1}}=-22.0857363551641+8.42266800899579 i$ & $\begin{array}{l}\widetilde{c}_{1_{1} 5_{2}} \\
0.180\end{array}$ & $\begin{array}{l}\tilde{c}_{1_{1} 5_{3}} \\
-9.4\end{array}$ \\
\hline$\tilde{c}_{1_{1} 6_{1}}=9.43611799993107-10.1165377535518 i$ & $\begin{array}{l}\tilde{c}_{1_{16} 6_{2}}= \\
-0.1524204278\end{array}$ & $\begin{array}{l}\tilde{c}_{1_{163}}= \\
14.9968021182233-1\end{array}$ \\
\hline$\tilde{c}_{1_{1} 7_{1}}=-23.2217879987250-18.5353612023957 i$ & $\begin{array}{l}\tilde{c}_{1_{1} 7_{2}}= \\
-1.84838342545925+19.3049333382765 i\end{array}$ & $\begin{array}{l}\tilde{c}_{1_{17}}= \\
46.7641650534058-30.5168801\end{array}$ \\
\hline$\tilde{c}_{1_{1} 8_{1}}=13.1129478953285+10.3385379254891 i$ & $\begin{array}{l}\tilde{c}_{1_{1} 8_{2}}= \\
4.54841895551983-12.4182935781109 i\end{array}$ & $\begin{array}{l}\tilde{c}_{1_{1} 8_{3}}= \\
-18.3079647571629-26.2365863851191 i\end{array}$ \\
\hline$\tilde{c}_{1_{1} 9_{1}}=-10.8370296923116-2.65335182564432 i$ & $\begin{array}{l}\tilde{c}_{1_{1} 9_{2}}= \\
-7.02131492970946+7.35188328378729 i\end{array}$ & $\begin{array}{l}\tilde{c}_{1_{1} 9_{3}}= \\
2.34858705035471+18.5759374713535 i\end{array}$ \\
\hline
\end{tabular}

of three concentric rings as shown in Figure 1. Antenna element distribution on each ring and the radii of rings are given in Table 1 . The radius of each half-wave dipole is considered to be equal to $5 \times 10^{-3} \lambda$ at an operating frequency of $2.4 \mathrm{GHz}$ and a $50 \Omega$ load is assumed to be connected to the terminal of each of the dipole antenna. The sources of EM radiation to be localised are modelled using three vertically polarised thin dipole antennas which are located at $\left(2.2 \lambda, 120^{\circ}, 40^{\circ}\right),\left(3 \lambda, 70^{\circ}, 70^{\circ}\right)$, and $\left(4 \lambda, 80^{\circ}, 20^{\circ}\right)$ which lie within the radiating near field of the receiver antenna array. Each of the source dipole is excited with a voltage source. Induced current at every dipole element of CCRA due to the incident field emanated from three dipole sources is computed by using the full-wave electromagnetic simulation package FEKO [27]. The antenna terminal voltage due to the received field is calculated as $V_{m}=Z_{L} I_{m}$ where this terminal voltage represents (17). Since terminal voltage measured at every antenna element in the array includes the contribution of the mutual coupling, the proposed compensation method is applied to compensate the effect of mutual coupling. A single (first) row of estimated compensation matrix which includes the elements $\widetilde{c}_{1_{1} 1_{1}}$ to $\widetilde{c}_{1_{1} 9_{3}}$ using proposed method is tabulated in Table 2.

5.1. Performance of CCRA for Different Arrangements of Antenna Elements. In order to analyse the effect of mutual coupling on the near-field parameter estimation using CCRA and chose optimum array configuration, CRLB for different arrangements of CCRA are calculated in the presence of mutual coupling as discussed earlier. Here, we consider four different arrangements of ring radii and element distributions for forming an optimum CCRA as given in Table 1. In every CCRA configuration under consideration, only three concentric rings are considered and the total number of dipole antenna elements in all rings put together is equal to 27. The antenna elements are placed uniformly on the circumference of the each ring. In order to derive CRLB for all the four cases, a single transmitting half-wave dipole is considered to be positioned at a known location of $\left(4 \lambda, 80^{\circ}, 20^{\circ}\right)$. The CRLB for estimated azimuth angle, elevation angle, and range for these cases are plotted in Figures 2-4, respectively. It can be observed, from Figures $2-4$, that the array configuration of case (d) has the lowest CRLB when compared to other cases (a), (b), and (c) and can be considered as an optimal configuration. Hence, we choose the optimal CCRA configuration given in case (d) for demonstrating compensation and parameter estimation.

5.2. Near-Field Parameter Estimation. Here, MUSIC and ESPRIT algorithms are applied for parameter estimation of near-field sources using the optimal CCRA configuration. Zero mean white Gaussian noise is added to the received signal at every element of CCRA. We apply the beamspace transformation on the array manifold of CCRA to map 


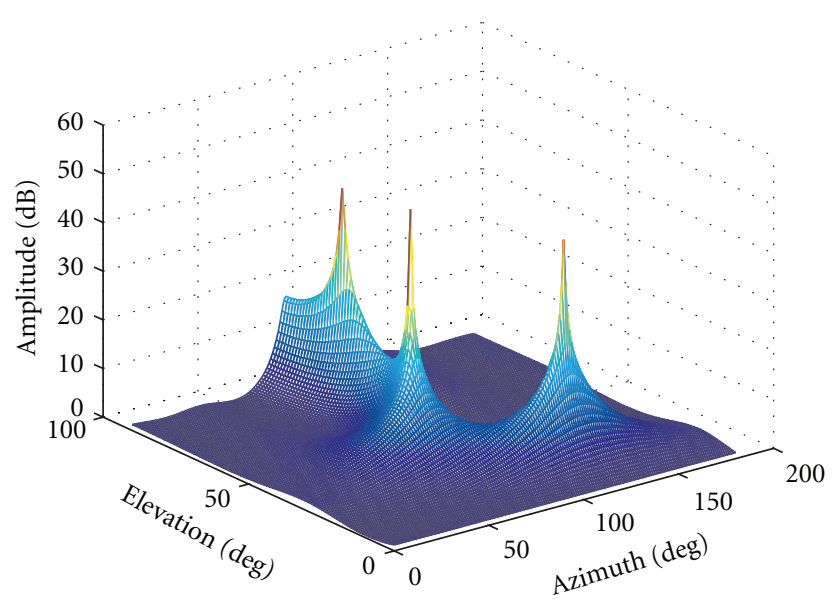

FIGURE 5: MUSIC pseudospectrum for ideal case where the effect of mutual coupling is ignored.

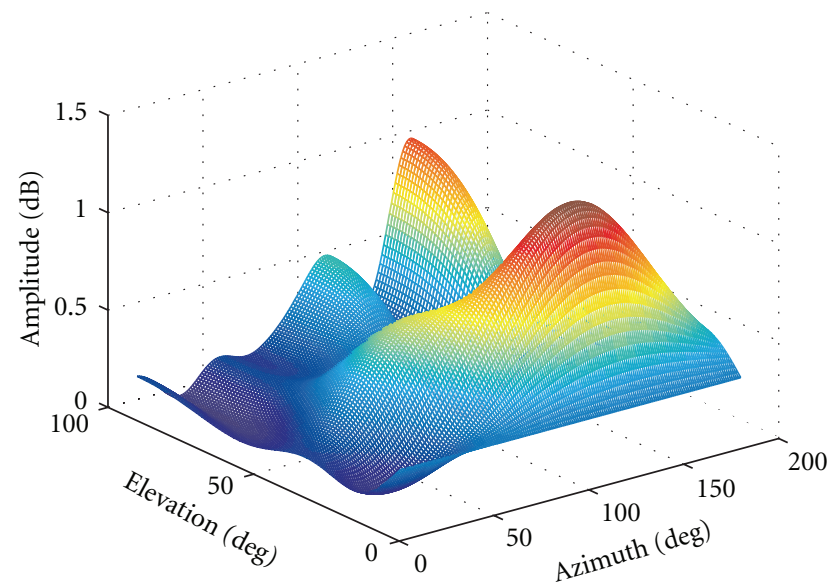

FIgURE 6: MUSIC pseudospectrum when mutual coupling is uncompensated.

it to an equivalent virtual linear array. The decorrelation technique available in [26] is applied since we consider the received signals to be correlated. For an idealised situation, that is, when the effect of mutual coupling is ignored, the estimated azimuth and elevation angles are shown by their pseudospectra in Figure 5. When the effect of mutual coupling is taken into consideration, without adopting any compensation, MUSIC pseudospectrum fails to create sharp peaks as shown in Figure 6. After applying the proposed compensation method, improved accuracy of parameter estimation can be observed from the peaks of MUSIC pseudospectra as shown in Figure 7. Similarly, in the presence of mutual coupling, that is, when no compensation is applied, ESPRIT algorithm also fails to estimate azimuth and elevation angles properly as shown in histogram plots of Figures 8 and 9, respectively. However, after applying the proposed compensation method, the estimation accuracy of azimuth and elevation angles using ESPRIT improves significantly as shown in histogram plots of Figures 10 and 11, respectively. The estimated ranges for near-field

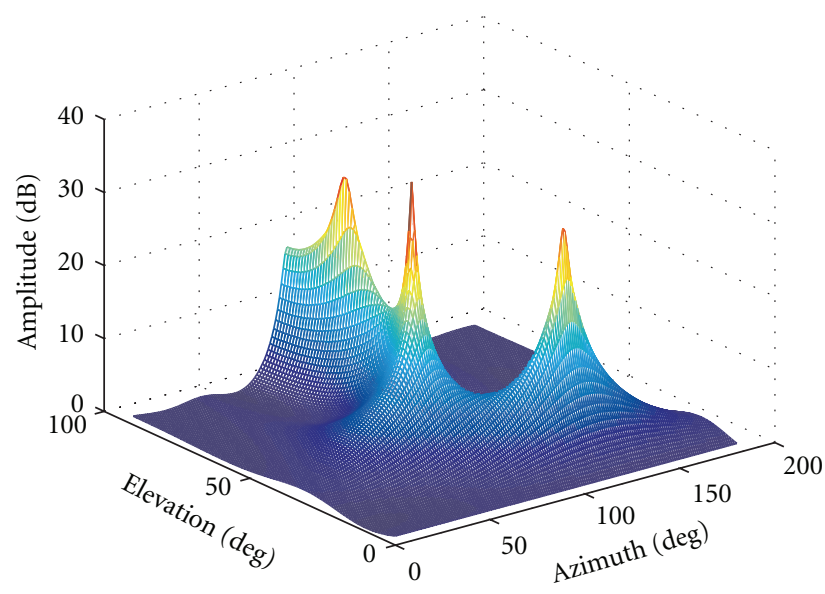

FIGURE 7: MUSIC pseudospectrum after the proposed compensation.

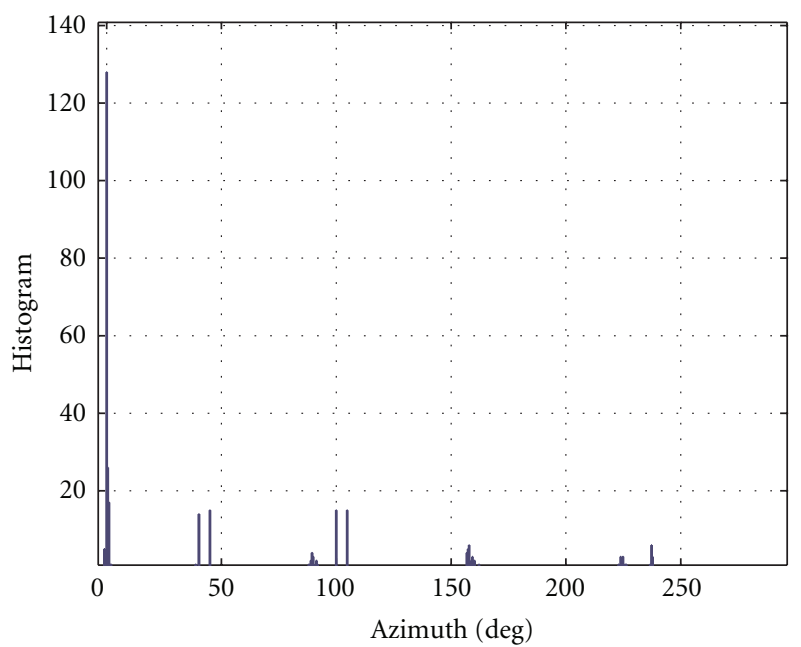

Figure 8: Histogram plot of estimated azimuth angles using ESPRIT algorithm when mutual coupling is uncompensated.

sources are calculated from the estimated time delay which is obtained by using cross-correlation among eigenvectors of signal subspaces. The coefficients of cross-correlations before compensating the effect of mutual coupling are plotted in Figure 12 whereas Figure 13 shows coefficients of cross-correlation after applying the proposed compensation method. Comparing Figures 12 and 13, it is obvious that, after the compensation, the peaks have become distinct and clearer. The optimal CCRA configuration, as explained earlier, is chosen for testing the performance of both MUSIC and ESPRIT algorithms, and their performance is plotted in Figures 14, 15, and 16 along with CRLB. It can be observed from these results that the MUSIC algorithm performs slightly better at lower SNRs when compared to ESPRIT. The RMSE of cross-correlation-based range estimation method is closer CRLB at higher SNRs. 


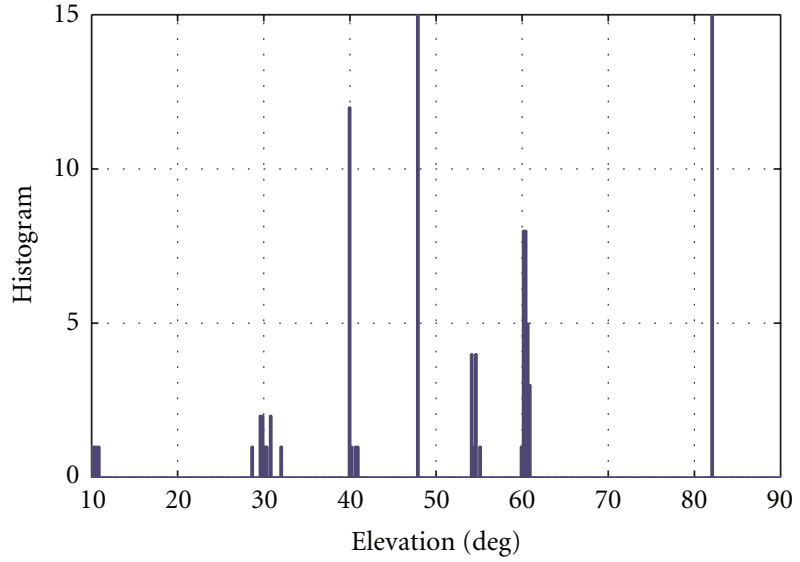

Figure 9: Histogram plot of estimated elevation angles using ESPRIT algorithm when mutual coupling is uncompensated.

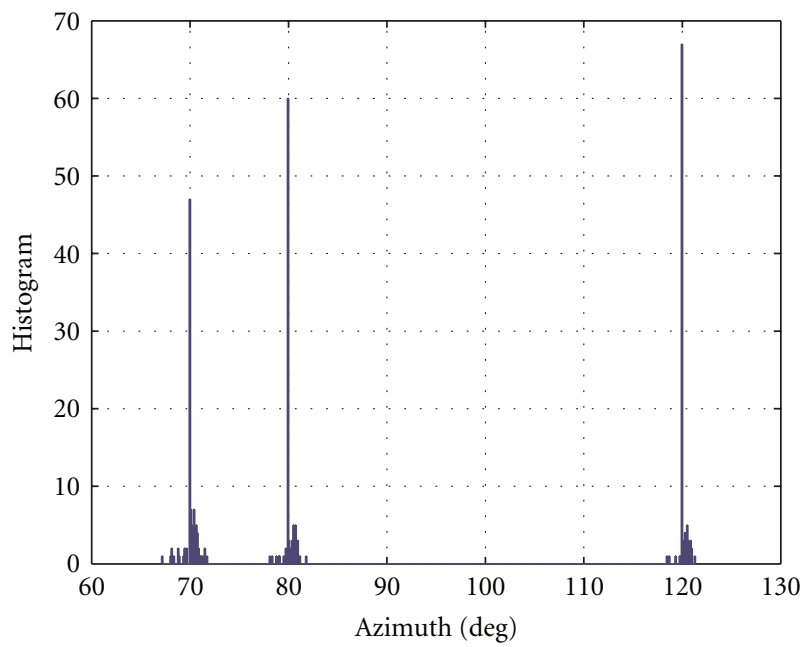

FIGURE 10: Histogram plot of estimated azimuth angles using ESPRIT algorithm after applying proposed compensation.

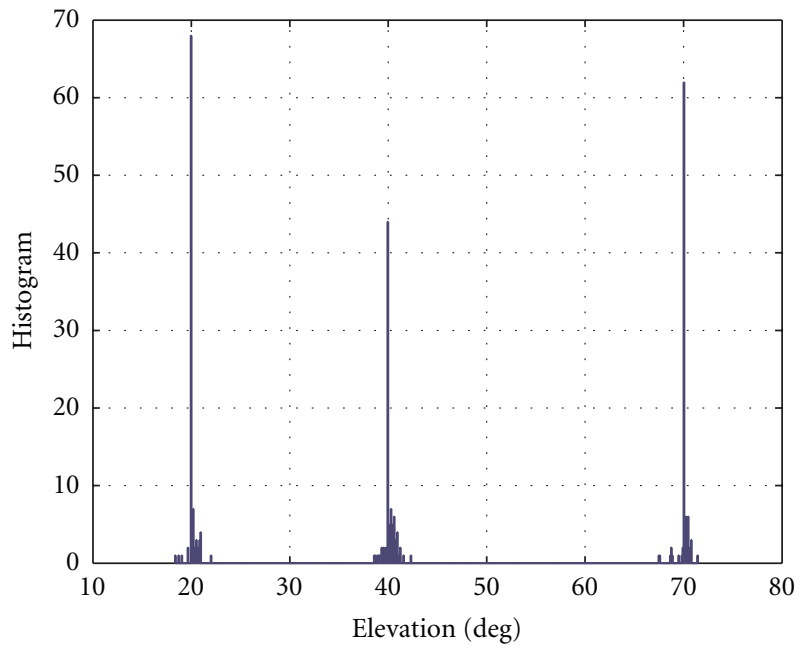

Figure 11: Histogram plot of estimated elevation angles using ESPRIT algorithm after applying proposed compensation.

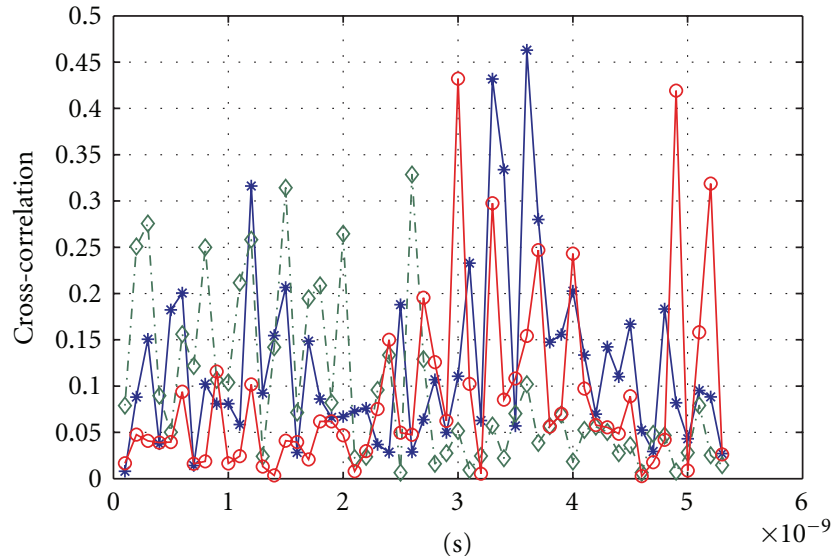

FIGURE 12: The cross-correlation among the eigenvectors of signal subspace when mutual coupling is uncompensated.

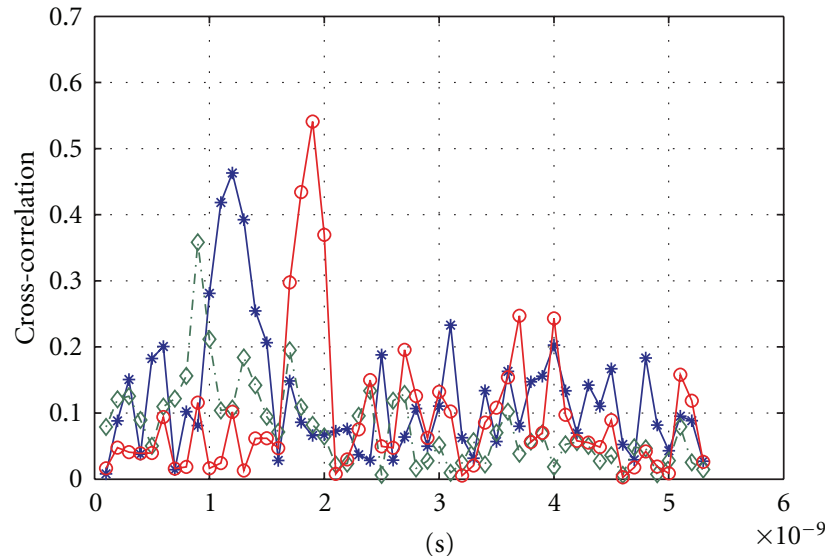

FIGURE 13: The cross-correlation among the eigenvectors of signal subspace after applying proposed compensation.

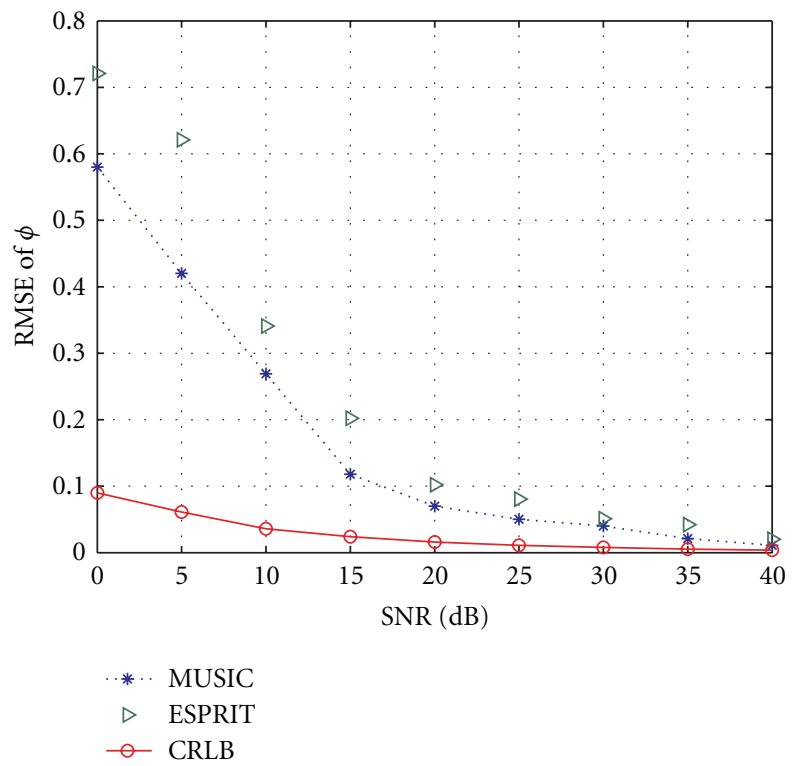

FIGURE 14: Performance comparison for the estimation of azimuth angle using MUSIC and ESPRIT algorithms for CCRA design of case (d). 


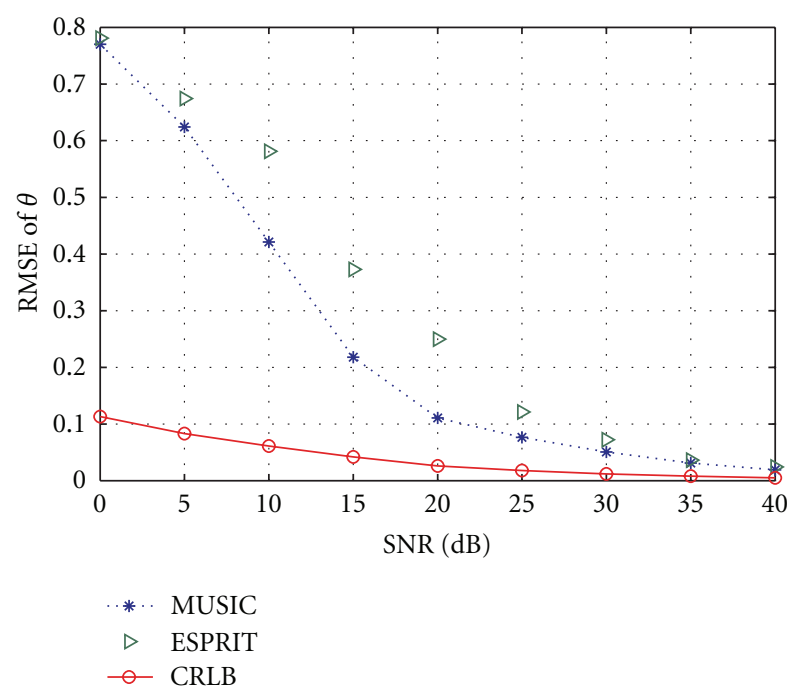

FIGURE 15: Performance comparison for the estimation of elevation angle using MUSIC and ESPRIT algorithms using CCRA design of case (d).

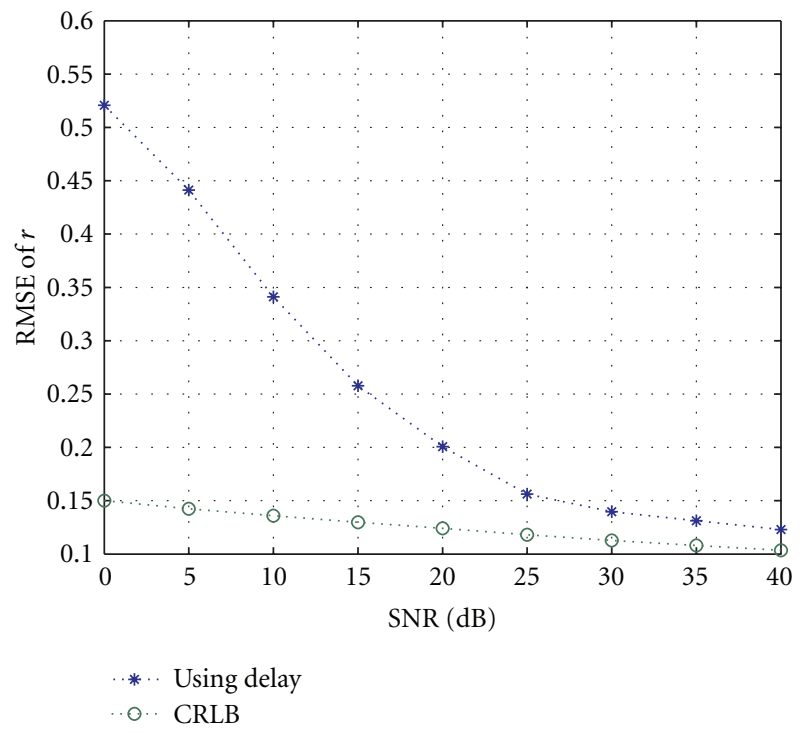

FIGURE 16: Performance of range estimation using cross-correlation based time-delay estimation method.

\section{Conclusions}

A mutual coupling compensation technique that is applicable with subspace-based super-resolution estimation algorithms is proposed in this paper. Concentric circular ring arrays are employed for testing the proposed method of mutual coupling compensation by estimating the nearfield parameters of transmitting dipoles using MUSIC and ESPRIT algorithms. In order to achieve a better accuracy on parameter estimation, an optimal CCRA configuration is chosen to obtain lowest CRLB in the presence of mutual coupling. The proposed compensation method is applied with MUSIC and ESPRIT algorithms for estimating azimuth and elevation angles, and the range parameter is estimated from the time delay obtained from the cross-correlation of signal subspace eigenvectors so as to avoid parameter search over $3 \mathrm{D}$ space and reduce the associated computational burden. The estimation performance after applying the proposed compensation method of mutual coupling is compared with CRLB for different SNRs. From simulation results, it can be observed that the proposed compensation method can effectively remove the effect of mutual coupling in CCRA and helps to improve the estimation performance of subspace-based algorithms in the presence of array mutual coupling.

\section{Appendix}

Elements of Fisher information matrix (FIM) (14) can be obtained as

$$
\begin{aligned}
& F_{\theta \theta}=2 N \operatorname{Re}\left\{\left(R_{s} \bar{A}^{H} R_{x}^{-1} A_{\theta}^{\prime}\right) \odot\left(R_{s} \bar{A}^{H} R_{x}^{-1} A_{\theta}^{\prime}\right)^{T}\right. \\
& \left.+\left(R_{s} \bar{A}^{H} R_{x}^{-1} \bar{A} R_{s}\right) \odot\left(\bar{A}_{\theta}^{H} R_{x}^{-1} A_{\theta}^{\prime}\right)^{T}\right\}, \\
& F_{\phi \phi}=2 N \operatorname{Re}\left\{\left(R_{s} \bar{A}^{H} R_{x}^{-1} A_{\phi}^{\prime}\right) \odot\left(R_{s} \bar{A}^{H} R_{x}^{-1} A_{\phi}^{\prime}\right)^{T}\right. \\
& \left.+\left(R_{s} \bar{A}^{H} R_{x}^{-1} \bar{A} R_{s}\right) \odot\left(\bar{A}_{\phi}^{H} R_{x}^{-1} A_{\phi}^{\prime}\right)^{T}\right\}, \\
& F_{r r}=2 N \operatorname{Re}\left\{\left(R_{s} \bar{A}^{H} R_{x}^{-1} A_{r}^{\prime}\right) \odot\left(R_{s} \bar{A}^{H} R_{x}^{-1} A_{r}^{\prime}\right)^{T}\right. \\
& \left.+\left(R_{s} \bar{A}^{H} R_{x}^{-1} \bar{A} R_{s}\right) \odot\left(\bar{A}_{r}^{H} R_{x}^{-1} A_{r}^{\prime}\right)^{T}\right\}, \\
& F_{\theta \theta}=2 N \operatorname{Re}\left\{\left(R_{s} \bar{A}^{H} R_{x}^{-1} A_{\theta}^{\prime}\right) \odot\left(R_{s} \bar{A}^{H} R_{x}^{-1} A_{\theta}^{\prime}\right)^{T}\right. \\
& \left.+\left(R_{s} \bar{A}^{H} R_{x}^{-1} \bar{A} R_{s}\right) \odot\left(\bar{A}^{H} R_{x}^{-1} A_{\theta}^{\prime}\right)^{T}\right\}, \\
& F_{\theta \phi}=2 N \operatorname{Re}\left\{\left(R_{s} A^{H} R_{x}^{-1} A_{\theta}^{\prime}\right) \odot\left(R_{s} A^{H} R_{x}^{-1} A_{\phi}^{\prime}\right)^{T}\right. \\
& \left.+\left(R_{s} A^{H} R_{x}^{-1} A_{\theta} R_{s}\right) \odot\left(A^{\prime}{ }_{\theta}^{H} R_{x}^{-1} A_{\phi}^{\prime}\right)^{T}\right\}, \\
& F_{\phi \theta}=2 N \operatorname{Re}\left\{\left(R_{s} A^{H} R_{x}^{-1} A_{\phi}^{\prime}\right) \odot\left(R_{s} A^{H} R_{x}^{-1} A_{\theta}^{\prime}\right)^{T}\right. \\
& \left.+\left(R_{s} A^{H} R_{x}^{-1} A_{\theta} R_{s}\right) \odot\left(A_{\phi}^{\prime H} R_{x}^{-1} A_{\theta}^{\prime}\right)^{T}\right\}, \\
& F_{r \phi}=2 N \operatorname{Re}\left\{\left(R_{s} A^{H} R_{x}^{-1} A_{\phi}^{\prime}\right) \odot\left(R_{s} A^{H} R_{x}^{-1} A_{r}^{\prime}\right)^{T}\right. \\
& \left.+\left(R_{s} A^{H} R_{x}^{-1} A_{r} R_{s}\right) \odot\left(A_{\phi}^{\prime H} R_{x}^{-1} A_{r}^{\prime}\right)^{T}\right\}, \\
& F_{r \theta}=2 N \operatorname{Re}\left\{\left(R_{s} A^{H} R_{x}^{-1} A_{\theta}^{\prime}\right) \odot\left(R_{s} A^{H} R_{x}^{-1} A_{r}^{\prime}\right)^{T}\right. \\
& \left.+\left(R_{s} A^{H} R_{x}^{-1} A_{\theta} R_{s}\right) \odot\left(A_{r}^{\prime H} R_{x}^{-1} A_{\theta}^{\prime}\right)^{T}\right\},
\end{aligned}
$$




$$
\begin{aligned}
& F_{\phi r}=2 N \operatorname{Re}\left\{\left(R_{s} A^{H} R_{x}^{-1} A_{r}^{\prime}\right) \odot\left(R_{s} A^{H} R_{x}^{-1} A_{\phi}^{\prime}\right)^{T}\right. \\
& \left.+\left(R_{s} A^{H} R_{x}^{-1} A_{r} R_{s}\right) \odot\left(A_{\phi}^{\prime H} R_{x}^{-1} A_{r}^{\prime}\right)^{T}\right\}, \\
& F_{\theta r}=2 N \operatorname{Re}\left\{\left(R_{s} A^{H} R_{x}^{-1} A_{r}^{\prime}\right) \odot\left(R_{s} A^{H} R_{x}^{-1} A_{\theta}^{\prime}\right)^{T}\right. \\
& \left.+\left(R_{s} A^{H} R_{x}^{-1} A_{\theta} R_{s}\right) \odot\left(A_{r}^{\prime}{ }_{r}^{H} R_{x}^{-1} A_{\theta}^{\prime}\right)^{T}\right\}, \\
& F_{\theta \alpha_{k}}=2 N \operatorname{Re}\left\{\left(R_{s} A^{H} R_{x}^{-1} A_{\alpha_{k}}^{\prime}\right) \odot\left(R_{s} A^{H} R_{x}^{-1} A_{\theta}^{\prime}\right)^{T}\right. \\
& \left.+\left(R_{s} A^{H} R_{x}^{-1} A_{\theta} R_{s}\right) \odot\left(A_{\alpha_{k}}^{\prime H} R_{x}^{-1} A_{\theta}^{\prime}\right)^{T}\right\}, \\
& F_{\theta \beta_{k}}=2 N \operatorname{Re}\left\{\left(R_{s} A^{H} R_{x}^{-1} A_{\beta_{k}}^{\prime}\right) \odot\left(R_{s} A^{H} R_{x}^{-1} A_{\theta}^{\prime}\right)^{T}\right. \\
& \left.+\left(R_{s} A^{H} R_{x}^{-1} A_{\theta} R_{s}\right) \odot\left(A_{\beta_{k}}^{\prime H} R_{x}^{-1} A_{\theta}^{\prime}\right)^{T}\right\}, \\
& F_{\phi \alpha_{k}}=2 N \operatorname{Re}\left\{\left(R_{s} A^{H} R_{x}^{-1} A_{\alpha_{k}}^{\prime}\right) \odot\left(R_{s} A^{H} R_{x}^{-1} A_{\phi}^{\prime}\right)^{T}\right. \\
& \left.+\left(R_{s} A^{H} R_{x}^{-1} A_{\phi} R_{s}\right) \odot\left(A_{\alpha_{k}}^{\prime H} R_{x}^{-1} A_{\phi}^{\prime}\right)^{T}\right\}, \\
& F_{\phi \beta_{k}}=2 N \operatorname{Re}\left\{\left(R_{s} A^{H} R_{x}^{-1} A_{\beta_{k}}^{\prime}\right) \odot\left(R_{s} A^{H} R_{x}^{-1} A_{\phi}^{\prime}\right)^{T}\right. \\
& \left.+\left(R_{s} A^{H} R_{x}^{-1} A_{\phi} R_{s}\right) \odot\left(A_{\beta_{k}}^{\prime H} R_{x}^{-1} A_{\phi}^{\prime}\right)^{T}\right\}, \\
& F_{r \alpha_{k}}=2 N \operatorname{Re}\left\{\left(R_{s} A^{H} R_{x}^{-1} A_{\alpha_{k}}^{\prime}\right) \odot\left(R_{s} A^{H} R_{x}^{-1} A_{r}^{\prime}\right)^{T}\right. \\
& \left.+\left(R_{s} A^{H} R_{x}^{-1} A_{r} R_{s}\right) \odot\left(A_{\alpha_{k}}^{\prime H} R_{x}^{-1} A_{r}^{\prime}\right)^{T}\right\}, \\
& F_{r \beta_{k}}=2 N \operatorname{Re}\left\{\left(R_{s} A^{H} R_{x}^{-1} A_{\beta_{k}}^{\prime}\right) \odot\left(R_{s} A^{H} R_{x}^{-1} A_{r}^{\prime}\right)^{T}\right. \\
& \left.+\left(R_{s} A^{H} R_{x}^{-1} A_{r} R_{s}\right) \odot\left(A_{\beta_{k}}^{\prime H} R_{x}^{-1} A_{r}^{\prime}\right)^{T}\right\} .
\end{aligned}
$$

\section{Acknowledgment}

The work reported in this paper is supported by the Australian Research Council through a Discovery Project Grant DP 0773234.

\section{References}

[1] I. J. Gupta and A. A. Ksienski, "Effect of mutual coupling on the performance of adaptive arrays," IEEE Transactions on Antennas and Propagation, vol. 31, no. 5, pp. 785-791, 1983.

[2] H. T. Hui, "Improved compensation for the mutual coupling effect in a dipole array for direction finding," IEEE Transactions on Antennas and Propagation, vol. 51, no. 9, pp. 2498-2503, 2003.

[3] T. T. Zhang, H. T. Hui, and Y. L. Lu, "Compensation for the mutual coupling effect in the ESPRIT direction finding algorithm by using a more effective method," IEEE Transactions on Antennas and Propagation, vol. 53, no. 4, pp. 1552-1555, 2005.

[4] T. T. Zhang, Y. L. Lu, and H. T. Hui, "Compensation for the mutual coupling effect in uniform circular arrays for 2D DOA estimations employing the maximum likelihood technique," IEEE Transactions on Aerospace and Electronic Systems, vol. 44, no. 3, pp. 1215-1221, 2008.

[5] K. R. Dandekar, H. Ling, and G. Xu, "Experimental study of mutual coupling compensation in smart antenna applications," IEEE Transactions on Wireless Communications, vol. 1, no. 3, pp. 480-487, 2002.

[6] R. S. Adve and T. K. Sarkar, "Compensation for the effects of mutual coupling on direct data domain adaptive algorithms," IEEE Transactions on Antennas and Propagation, vol. 48, no. 1, pp. 86-94, 2000.

[7] H. Rogier and D. De Zutter, "Beamforming strategies for compact arrays in mobile terminals using the exact active element pattern method," Microwave and Optical Technology Letters, vol. 35, no. 3, pp. 201-203, 2002.

[8] C. K. E. Lau, R. S. Adve, and T. K. Sarkar, "Minimum norm mutual coupling compensation with applications in direction of arrival estimation," IEEE Transactions on Antennas and Propagation, vol. 52, no. 8, pp. 2034-2041, 2004.

[9] Q. Yuan, Q. Chen, and K. Sawaya, "Accurate DOA estimation using array antenna with arbitrary geometry," IEEE Transactions on Antennas and Propagation, vol. 53, no. 4, pp. 13521357, 2005.

[10] A. J. Weiss and B. Friedlander, "DOA and steering vector estimation using a partially calibrated array," IEEE Transactions on Aerospace and Electronic Systems, vol. 32, no. 3, pp. 1047-1057, 1996.

[11] E. K. L. Hung, "Matrix-construction calibration method for antenna arrays," IEEE Transactions on Aerospace and Electronic Systems, vol. 36, no. 3, pp. 819-828, 2000.

[12] Q. Bao, C. C. Ko, and W. Zhi, "DOA estimation under unknown mutual coupling and multipath," IEEE Transactions on Aerospace and Electronic Systems, vol. 41, no. 2, pp. 565-573, 2005.

[13] S. Kikuchi, H. Tsuji, and A. Sano, "Autocalibration algorithm for robust Capon beamforming," IEEE Antennas and Wireless Propagation Letters, vol. 5, no. 1, Article ID 874070, pp. 251255, 2006.

[14] B. Friedlander and A. J. Weiss, "Direction finding in the presence of mutual coupling," IEEE Transactions on Antennas and Propagation, vol. 39, no. 3, pp. 273-284, 1991.

[15] H. S. Lui and H. T. Hui, "Improved mutual coupling compensation in compact antenna arrays," IET Microwaves, Antennas and Propagation, vol. 4, no. 10, pp. 1506-1516, 2010.

[16] H. S. Lui, H. T. Hui, and M. S. Leong, "A note on the mutualcoupling problems in transmitting and receiving antenna arrays," IEEE Antennas and Propagation Magazine, vol. 51, no. 5, Article ID 5432083, pp. 171-176, 2009.

[17] H. S. Lui and H. T. Hui, "Mutual coupling compensation for direction-of-arrival estimations using the receiving-mutualimpedance method," International Journal of Antennas and Propagation, vol. 2010, Article ID 373061, 7 pages, 2010.

[18] C. P. Mathews and M. D. Zoltowski, "Eigenstructure techniques for 2-D angle estimation with uniform circular arrays," IEEE Transactions on Signal Processing, vol. 42, no. 9, pp. 23952407, 1994.

[19] M. D. Zoltowski, M. Haardt, and C. P. Mathews, "Closed-form 2-D angle estimation with rectangular arrays in element space 
or beamspace via unitary ESPRIT," IEEE Transactions on Signal Processing, vol. 44, no. 2, pp. 316-328, 1996.

[20] R. L. Haupt, "Optimized element spacing for low sidelobe concentric ring arrays," IEEE Transactions on Antennas and Propagation, vol. 56, no. 1, pp. 266-268, 2008.

[21] Z. Ye and C. Liu, "2-D DOA estimation in the presence of mutual coupling," IEEE Transactions on Antennas and Propagation, vol. 56, no. 10, pp. 3150-3158, 2008.

[22] P. Stoica and A. Nehorai, "MUSIC, maximum likelihood, and Cramer-Rao bound," IEEE Transactions on Acoustics, Speech, and Signal Processing, vol. 37, no. 5, pp. 720-741, 1989.

[23] T. Svantesson, "Modeling and estimation of mutual coupling in a uniform linear array of dipoles," in Proceedings of the IEEE International Conference on Acoustics, Speech, and Signal Processing (ICASSP '99), pp. 2961-2964, Phoenix, Ariz, USA, March 1999.

[24] T. Huang and A. S. Mohan, "Effects of array mutual coupling on near-field DOA estimation," in Proceedings of the Canadian Conference on Electrical and Computer Engineering: Toward a Caring and Humane Technology (CCECE '03), pp. 1881-1884, May 2003.

[25] S. H. Lee, C. S. Ryu, and K. K. Lee, "Near-field source localisation using bottom-mounted linear sensor array in multipath environment," IEE Proceedings: Radar, Sonar and Navigation, vol. 149, no. 4, pp. 202-206, 2002.

[26] C. P. Mathews and M. D. Zoltowski, "Performance analysis of the UCA-ESPRIT algorithm for circular ring arrays," IEEE Transactions on Signal Processing, vol. 42, no. 9, pp. 2535-2539, 1994.

[27] FEKO EM Software and Systems S.A. (Pty) Ltd, 32 Techno Lane, Technopark, Stellenbosch, 7600, South Africa. 

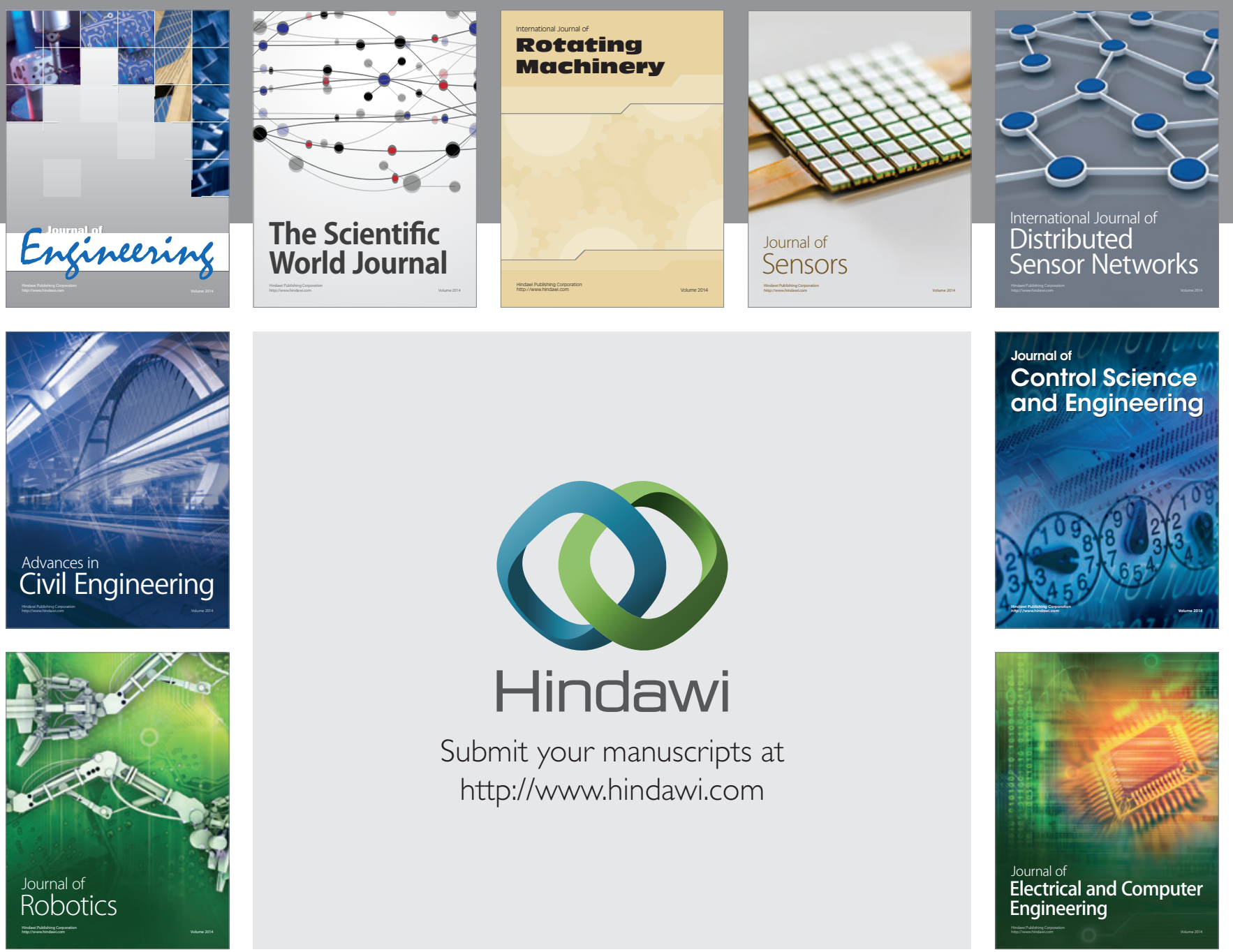

Submit your manuscripts at

http://www.hindawi.com
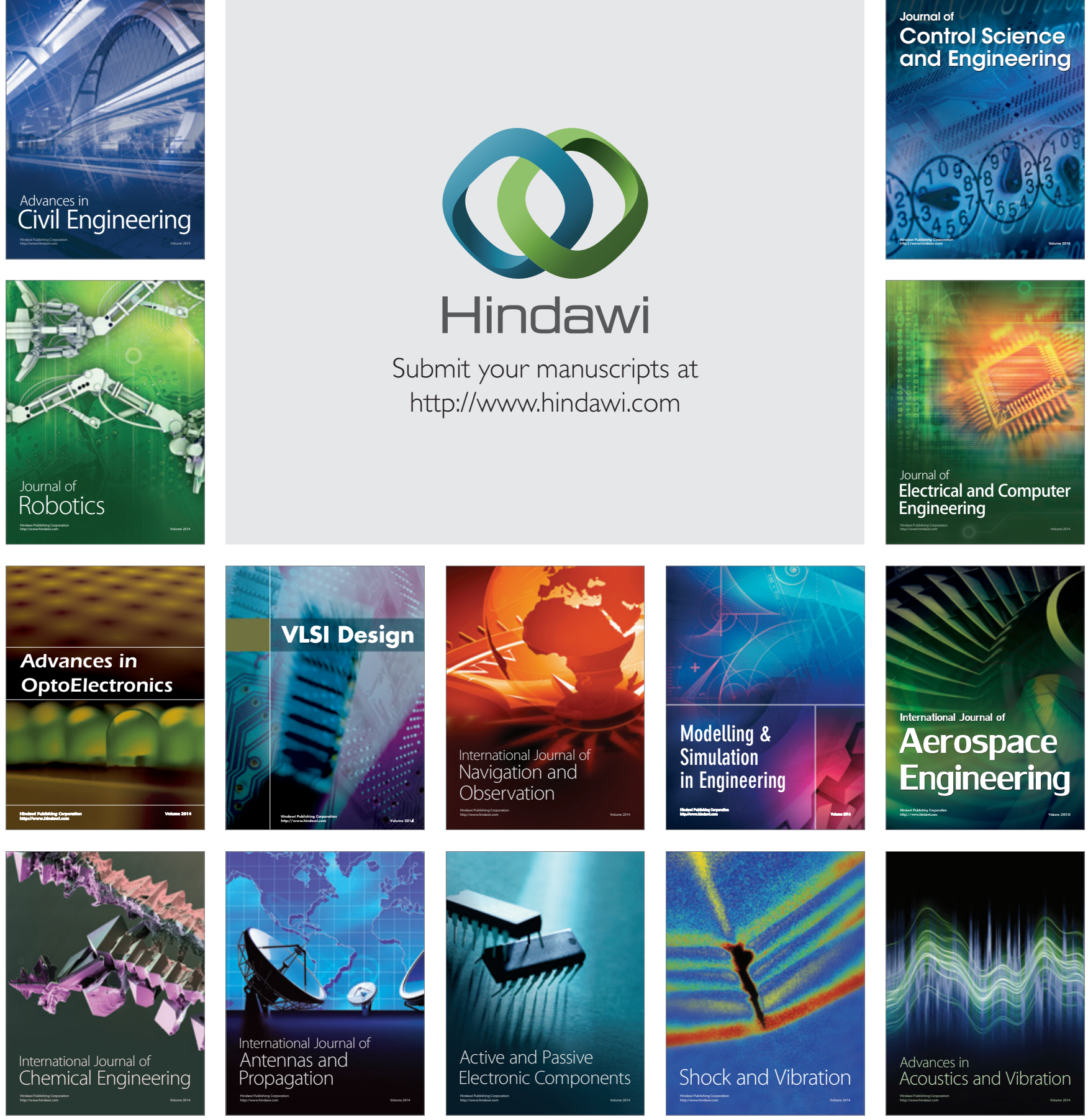